pour transfecter de nouvelles cultures de cellules $\mathrm{NIH}_{3} \mathrm{~T}_{3}$. Des foyers apparaissent à nouveau, dus aussi au transfert du gène transformant humain. Une banque d'ADN génomique est alors constituée à partir de ces foyers secondaires (voir médecine/sciences $n^{\circ}$ 6, vol. 2, p. 333) et est criblée avec des séquences d'ADN répétitives dans le génome humain, mais absentes du génome de souris. Le fragment d'ADN humain, candidat pour être responsable de l'acquisition du phénotype malin, est alors isolé et sa structure peut être caractérisée (figure I). Son pouvoir transformant peut aussi être confirmé par une troisième expérience de transfection de cellules $\mathrm{NIH}_{3} \mathrm{~T}_{3}$ à l'aide de cet ADN cloné. Dans l'exemple de l'ADN d'un cancer du col utérin étudié ici, le gène transformant se révéla contenir tout ou partie du génome d'un papillomavirus type I6. Un fragment de cet ADN viral utilisé comme sonde permettait de détecter dans les cellules transformées des ARN de I,8 (forme prédominante) à $6,8 \mathrm{~kb}$. Un résultat similaire avait été rapporté l'an dernier par l'équipe de Zur Hausen: des ARN hybridant avec des sondes virales type 16 étaient détectés dans des biopsies de cancer du col utérin [5]. Il apparaît donc que les papillomavirus type 16 et 18 , comme les rétrovirus HTLV I et 2 responsables de leucémies humaines, sont les agents directs de cancers humains, étendant donc le nombre des tumeurs malignes viroinduites chez l'homme. Dans ce cas, il s'agit de plus d'une infection sexuellement transmissible, comme le suggère fortement la présence de certains types de papillomarivus dans le sperme d'hommes ayant des condylomes génitaux [6].

Rappelons que dans d'autres cancers humains associés à une infection virale par le virus de l'hépatite B ou par le virus d'Epstein Barr par exemple, le mécanisme de la cancé- risation procéderait plutôt d'une interaction entre le génome viral et l'ADN de la cellule infectée que de l'action directe d'un gène transformant viral. $\quad$ A. K.

I. Durst M, Gissmann L, Ikenberg $H$, Zur Hausen $H$. A papillomarivus DNA from a cervical carcinoma and its prevalence in cancer biopsy samples from different geographic regions. Proc Natl Acad Sci USA 1983; 80: 3812-5.

2. Boshart M, Gissmann L, Ikenberg H, Kleinhein A, Scheurlen W, Zur Hausen H. A new type of papillomavirus DNA, its presence in genital cancer biopsies and in cell lines derived from cervical cancer. EMBO J 1984; 3: 1 I 5 I-7.

3. Beaudenon S, Kremsdorf D, Croissant O, Jablonska $S$, Wain-Hobson $S$, Orth G. A novel type of human papillomavirus associated with genital neoplasias. Nature 1986; 321: 246-9.

4. Tsunokawa $Y$, Takebe $N$, Kasamatsu $T$, Terada M, Sugimura T. Transf orming activity of human papillomavirus type 16 DNA sequences in a cervical cancer. Proc Natl Acad Sci USA 1986; 83: 2200-3.

5. Schwartz E, Freese UK, Gissmann L, et al. Structure and transcription of human papillomavirus sequences in cervical carcinoma cells. Nature 1985; 314: II I-4.

6. Ostrow RS, Zachow KR, Niimura $M$ et al. Detection of papillomavirus DNA in human semen. Science 1986; 231: 731-3.

\title{
Une utilisation inédite des essais nucléaires: la datation des calculs biliaires
}

\begin{abstract}
Lorsque l'on découvre des calculs biliaires, on n'a le plus souvent aucun renseignement sur leur âge et la façon dont ils se sont développés depuis leur formation. Ce type d'information pourrait avoir une importance pour comprendre l'histoire naturelle de la lithiase et tenter d'identifier les causes de ses complications.

Mok et coll. [I] ont utilisé une méthode élégante pour dater les calculs biliaires chez des malades opérés. Ils ont mis à profit l'enrichissement de l'atmosphère en ${ }^{14} \mathrm{C}$ depuis les premiers essais thermonucléaires des années 1950-1960: ces explosions ont $e u$ pour résultat un doublement $d u$
\end{abstract}

${ }^{14} \mathrm{C}$ atmosphérique, par rapport à son taux antérieur, en 1963. Le ${ }^{14} \mathrm{C}$ ainsi produit a été rapidement oxydé en ${ }^{14} \mathrm{CO}_{2}$. Celui-ci a été utilisé par les végétaux, puis par les animaux qui l'ont incorporé avec un retard moyen d'environ deux ans. Il a été montré que le rapport $d u^{14} C$ au ${ }^{12} C$ (le carbone naturel) était virtuellement identique dans les composants de la "biosphère " et dans l'atmosphère, depuis I955. Cela permet de dater un échantillon biologique $\grave{a}$ I an I/2 à 2 ans près.

Les calculs de 15 malades ont ainsi été étudiés. Aucun n'avait eu d'examens utilisant un isotope radioactif et tous avaient eu des cholécystographies antérieurement. Onze malades avaient des symptômes en rapport avec leurs calculs et quatre étaient asymptomatiques. Les calculs (obtenus à l'intervention che $z$ I3 malades et à l'autopsie chez deux malades) ont été analysés par couches concentriques. Le $\mathrm{CO}_{2}$ de chaque couche de calculs a été obtenu par combustion, purifié sur une colonne de charbon activé et la radioactivité $d u{ }^{14} C$ mesurée par compteur à gaz proportionnel.

L'âge du calcul a été obtenu à partir $d u$ contenu en ${ }^{14} C d u$ calcul, au moyen d'une courbe de référence 
représentant Pactivité $d u{ }^{14} C d u$ sang, de cheveux et d'organes humains en fonction du temps depuis 1950. Au total, 59 échantillons de calculs ont été analysés. L'âge des calculs a été corrélé à la date du début des symptômes et à d'autres données cliniques. Les symptômes (ou les complications) de la lithiase ne sont jamais apparus moins de deux ans après la formation des calculs; ils sont apparus, en moyenne, huit ans après (écart-type: 5,I ans). Chez les malades opérés, la cholécystectomie a été faite en moyenne $I I, 7 \pm 4,6$ ans après la formation des calculs. Chez quatre malades, existaient des calculs de taille différente: chez deux d'entre eux, tous les calculs avaient le même âge; certains calculs avaient donc cessé de croître, tandis que d'autres avaient continué. A l'inverse, chez deux autres malades, les plus petits calculs étaient manifestement les plus jeunes. La vitesse de croissance des calculs était assez voisine pour tous et pratiquement identique chez les malades symptomatiques et les malades asymptomatiques $(2,6 \pm I, 4$ et 2,6士I,I mm par an). Enfin, des renseignements ont pu être obtenus pour deux calculs cholédociens. L'un avait le même âge que les calculs vésiculaires prélevés simultanément; Pautre, enlevé deux ans après la cholécystectomie, avait apparemment grossi dans la voie biliaire principale. Ces résultats sont encore fragmentaires. Ils démontrent cependant la possibilité de dater les calculs par cette méthode avec une assez grande précision. Appliquée à un plus grand nombre de malades, la méthode peut fournir de précieux renseignements sur l'histoire naturelle de la lithiase, avant qu'elle ne devienne symptomatique. La période de latence assez longue entre la formation des calculs et les premiers symptômes permet d'espérer que l'on puisse interrompre la progression de la maladie au moyen d'un traitement de dissolution.

\section{S. E.}

\footnotetext{
I. Mok HYI, Druffel ERM, Rampone WM Chronology of cholclithiasis. Dating gallstones from atmospheric radiocarbon produced by nuclear bomb explosions. $N$ Engl 7 Med 1986; 314: 1075-7.

$m / s n^{\circ} 7$, vol. 2, septembre 86
}

\title{
AMP cyclique. apprentissage et mémoire
}

\author{
La mutation dnc de la drosophile
}

Le gène dunce (dnc) de la drosophile semble contrôler de nombreux paramètres comportementaux dépendant de l'apprentissage. Ses mutations entraînent principalement une extraordinaire propension à l'oubli : les insectes peuvent être conditionnés mais oublient bien vite ce qu'ils ont appris. D'autres comportements plus instinctifs sont eux-mêmes modifiés, tels ceux des prémices nuptiaux [I].

Au point de vue biochimique, la principale anomalie observée chez les mutants semble être un déficit plus ou moins complet en l'activité d'une AMPc phosphodiesterase, enzyme qui dégrade l'AMP cyclique, et par conséquent une forte augmentation de la concentration intracellulaire en cette substance [2]. Ces constatations suggèrent fortement que, au moins chez la drosophile, l'AMP cyclique intervient dans les processus biochimiques qui sont à la base de l'apprentissage et de la mémoire.

L'étude de différents types de mutants ayant des délétions variables du chromosome $\mathrm{X}$ permit de localiser le gène dnc au niveau d'un fragment de 50 kilobases $(\mathrm{kb})$ de ce chromosome. L'utilisation de sites polymorphes pour la coupure par des enzymes de restriction conduisit, par une étude génétique de la liaison entre ces polymorphismes et la transmission du phénotype muté, à la localisation plus fine du gène au niveau d'un fragment de $12 \mathrm{~kb}$ [3]. Des clones génomiques contenant ce fragment furent ensuite obtenus et utilisés comme sondes pour analyser l'expression d'ARN messagers spécifiques au cours du développement. Le gène dnc semble être exprimé sous la forme d'au moins six transcrits différents, de 4,5 à $9,6 \mathrm{~kb}$, dont les concentrations varient considérablement selon les tissus et les stades de développement. Certains de ces transcrits sont qualitativement modifiés chez les mutants [3]. Une telle complexité est inattendue si l'on fait l'hypothèse que le gène dnc code seulement pour la phosphodiesterase déficiente chez les mutants. L'activité de cette enzyme au cours du développement des insectes normaux ne suit d'ailleurs pas fidèlement les modifications des $A R N m$ codés par le gène dnc [3].

Les liens existant entre le locus dunce, l'AMPc-phosphodiesterase, l'augmentation cellulaire en AMP cyclique et les troubles de la mémoire sont donc peut-être moins simples que ne semblaient l'indiquer de premières hypothèses. Il n'en reste pas moins que ce modèle génétique des drosophiles porteuses d'une mutation monogénique, ayant un trouble biochimique parfaitement caractérisé et des anomalies complexes portant sur les processus neurobiologiques de l'apprentissage et de la mémoire, pourrait bien constituer le moyen d'une "percée" des connaissances dans ce domaine si complexe.

A. $\mathbf{K}$.

1. Aceves-Pina EO, Booker R, Duerr JS, et al. Learning and memory in drosophila, studied with mutants. Cold Spring Harbor Symp Quant Biol 1984; 48: 831-40.

2. Davis RL, Kiger JA. Dunce mutants of drosophila mclanogaster: mutants defective in the cyclic AMP phosphodicsterase enzyme system. J Cell Biol 1981; 90: 101-7.

3. Davis RL, Davidson N. The memory gene dunce ${ }^{+}$encodes a remarkable set of RNAs with internal heterogeneity. Mol Cell Biol 1986; 6: 1464-70. 